\title{
COVID-19: USO Y ABUSO DE INTERNET Y TELÉFONO MÓVIL EN ESTUDIANTES UNIVERSITARIOS
}

\author{
Ma Villa Carpio-Fernández \\ Departamento de Psicología \\ Área de Psicología Evolutiva y de la Educación \\ Universidad de Jaén \\ mvcarpio@ujaen.es \\ Mà Cruz García-Linares \\ Departamento de Psicología \\ Área de Psicología Evolutiva y de la Educación \\ Universidad de Jaén \\ Mํㅡㄹ Teresa Cerezo-Rusillo \\ Departamento de Psicología \\ Área de Psicología Evolutiva y de la Educación \\ Universidad de Jaén \\ Pedro Félix Casanova-Arias \\ Departamento de Psicología \\ Área de Psicología Evolutiva y de la Educación \\ Universidad de Jaén
}

Recepción Artículo: 12 mayo 2021

Admisión Evaluación: 12 mayo 2021

Informe Evaluador 1: 18 mayo 2021

Informe Evaluador 2: 25 mayo 2021

Aprobación Publicación: 02 junio 2021

\section{RESUMEN}

La pandemia provocada por el COVID-19 ha provocado un cambio en los hábitos de vida de la población en general. En el último año, la tecnología ha constituido para el estudiante un recurso eficaz, no solo a nivel académico sino también social y familiar. Sin embargo, a pesar de la utilidad de las nuevas tecnologías, un uso inadecuado 0 abusivo puede producir consecuencias negativas en la vida familiar, social y académica del estudiante. El objetivo de este trabajo consiste en analizar los efectos que ha producido la pandemia provocada por el COVID-19 en los hábitos de jóvenes universitarios; uso de las nuevas tecnologías, tiempo diario dedicado al uso de internet y teléfono móvil, así como en el posible uso problemático de los mismos. La muestra estaba compuesta por 671 estudiantes universitarios de diferentes titulaciones de la Universidad de Jaén. Se utilizó una adaptación del cuestionario de Oliva et al. (2012) sobre el hábito de uso de las nuevas tecnologías. Las cuestiones hacen referencia a la edad de inicio, nivel de uso, tiempo diario de uso antes y después del COVID-19, tipo de actividades que realizan habitualmente en internet y el uso problemático de internet y el teléfono móvil en el ámbito académico, social y familiar. Los resultados indican que la COVID-19 ha provocado un incremento en el núme- 


\section{COVID-19: USO Y ABUSO DE INTERNET Y TELÉFONO MÓVIL EN ESTUDIANTES UNIVERSITARIOS}

ro de horas dedicadas al uso de internet y teléfono móvil. Las actividades para las que utilizan internet con más frecuencia son la realización de trabajos académicos, correo electrónico y redes sociales, chats o foros. Finalmente, aquellos que indican hacer un uso abusivo de internet y teléfono móvil, señalaban tener problemas académicos, sociales y familiares. Podemos concluir que, aunque se produce un aumento en el uso de las nuevas tecnologías como consecuencia del COVID-19, su uso, en estudiantes universitarios se destina mayormente a la formación académica, aunque también está provocando algunos problemas en los diferentes ámbitos de la vida de los estudiantes.

Palabras clave: COVID-19; estudiantes universitarios; internet; teléfono móvil

\section{ABSTRACT}

Covid-19: internet and cell phone use and abuse in university students. The COVID-19 pandemic has caused a change in the life habits of the general population. In the last year, technology has constituted an effective resource for the student, at an academic, social and family level. However, despite the usefulness of new technologies, inappropriate or abusive use can lead to negative consequences in the student's family, social and academic life. The objective of this work is to analyze the effects that the pandemic caused by COVID-19 has produced on the habits of young university students; use of new technologies, daily time dedicated to the use of the internet and mobile phone, as well as the possible problematic use of them. The simple consisted of 671 university students with different degrees from the University of Jaen. The questionnaire by Oliva et al., (2012) on the habit of using new technologies. The questions refer to the age of onset, level of use, daily time of use before and after COVID-19, type of activities that they habitually carry out on the internet and the problematic use of the internet and mobile phone in the academic, social and family. The results indicate that COVID-19 has caused an increase in the number of hours dedicated to using the internet and mobile phone. The activities for which they use the internet most frequently are academic work, email and social networks, chats or forums. Finally, among those who indicate abusive use of the internet and mobile phone, they indicated having academic, social and family problems. We can conclude that, although there is an increase in the use of new technologies as a result of COVID-19, their use in university students is mainly intended for academic training, although it is also causing some problems in different areas of life of the students.

Keywords: COVID-19; university students; internet; mobile phone

\section{INTRODUCCIÓN}

En los últimos años, el uso generalizado de las tecnologías de la información y la comunicación han tenido un importante impacto en la sociedad actual, principalmente entre la población más joven. Este dato unido a la situación de pandemia por la COVID-19 que venimos sufriendo ha provocado cambios significativos en el funcionamiento de la sociedad, no solo a nivel sanitario, económico y social sino también a nivel educativo. La situación actual que vivimos, confinamiento en el hogar o limitación de la movilidad, nos ha llevado a adoptar nuevos hábitos de vida, siendo la forma virtual la utilizada para acceder al trabajo, educación, contacto con familia y amigos, así como a las actividades de ocio. En esta situación, los estudiantes universitarios han tenido que poner en funcionamiento nuevas estrategias cognitivas, afectivas y motivacionales para autorregular su aprendizaje y poder adaptarse a una versión virtual de los estudios que hasta ahora realizaban presencialmente (Osorio et al. 2010).

Las posibilidades que ofrecen las nuevas tecnologías han dado lugar a nuevas formas de interacción social entre los jóvenes, convirtiéndose en una herramienta de comunicación social básica (Molero et al., 2014; Pintado y Sánchez, 2012). Ahora bien, esta nueva situación conlleva una preocupación social acerca de la influencia que estas tecnologías están teniendo en el desarrollo y ajuste personal de los adolescentes y jóvenes (Díaz-Vicario et al. 2019; Orte et al. 2020). Un uso moderado y adecuado de ellas puede ser positivo, pero el abuso puede provocar efectos negativos en el desarrollo cognitivo, afectivo, social y de identidad de este sector de la población (Alonso y Romero 2017; Arab y Díaz, 2015). 
Respecto a los efectos positivos, Torres et al. (2015) han encontrado relaciones positivas entre el uso de internet y el éxito académico en estudiantes universitarios. Asimismo, el empleo de las redes sociales facilita la comunicación, la socialización y la colaboración entre individuos que comparten determinadas características, intereses y/o necesidades (Espuny et al. 2011; Heyman, 2014). Con el teléfono móvil los jóvenes buscan información, expresan sentimientos e inquietudes, solucionan problemas y asuntos sentimentales que les permiten avanzar en el logro de su identidad personal (Oliva et al., 2012 y Besoli, 2018).

En cuanto a los posibles riesgos y principales preocupaciones, hay estudios que señalan el uso cada vez más temprano de estos dispositivos (Besoli, 2018) o el tiempo total diario dedicado por los jóvenes y adolescentes al teléfono móvil o al ordenador, que según los datos disponibles podrían representar gran parte de su actividad diaria (Díaz- Vicario et al. 2019; Echeburúa et al. 2009; Muñoz-Miralles et al., 2014).

Aunque en principio no tendría que preocupar el uso cada vez mayor de estas tecnologías, el problema se produce cuando afecta negativamente al desarrollo normal de la vida cotidiana del individuo (Oliva et al., 2012); descuidando actividades académicas (Haddon y Vicent, 2014) y disminuyendo el tiempo dedicado a la familia, amigos y ocio, por estar más tiempo en el "mundo virtual" que en el "real" (Diaz-Vicario et al., 2019), lo que puede llegar a crear situaciones de dependencia y pérdida de control derivadas de la necesidad de mantenerse socialmente activos. Según el estudio realizado por Martínez de Moretín et al. (2014) los jóvenes adoptan nuevas pautas de conducta que afectan a las relaciones personales y familiares, encontrándonos a adolescentes aislados que pasan gran parte de su tiempo en soledad. Ahora bien, no debemos confundir la dependencia con el sentirse atraído por las nuevas tecnologías (Oliva et al., 2012), existiendo dependencia cuando la tecnología pasa de ser un medio a constituir un fin en sí misma (Becoña, 2006).

El estudio realizado por Oliva et al., (2012) sobre el uso y riesgo de adicciones a las nuevas tecnologías entre adolescentes y jóvenes andaluces ha aportado datos sobre la edad de inicio, tiempo diario de uso, momento y tipo de actividades que los jóvenes realizan habitualmente, encontrando de forma general que la familiarización e incorporación a las nuevas tecnologías es cada más temprana y que la edad de inicio se retrasa según aumenta la edad de los sujetos, datos que coinciden con los encontrados en trabajos posteriores (Carpio et al. 2020).

Respecto al tiempo diario de uso, Oliva et al., (2012) encontraron que un tercio de los sujetos entrevistados, con edades comprendidas entre los 15 y 24 años, utilizaban estas tecnologías una media de 3 horas diarias. Estos resultados son acordes con otros estudios (Labrador y Villadango, 2009; Pérez-Díaz y Rodríguez, 2008) que señalan que el móvil, junto al ordenador son los dispositivos a los que los adolescentes dedican más tiempo diario. En cuanto al tipo de actividades Oliva et al., (2012) señalaron que los jóvenes utilizaban estas tecnologías para comunicarse socialmente, enviar mensajes, chats o foros, descargar películas y videos, redes sociales, tareas académicas, búsqueda de información sobre temas de interés y juegos online, siendo las más habituales el uso de las redes sociales y los chats o foros.

En los momentos actuales el tema de las tecnologías ha generado aún más interés debido al incremento significativo que se ha producido en su uso debido al estado de alarma en el que vivimos hace ya más de un año. Según estudios recientes, se ha relacionado con problemas de autocontrol, ansiedad, depresión, problemas sociales, trastornos del sueño y consecuentemente con problemas de rendimiento académico y falta de atención (Cerezo et al. 2020; Orte y Nevot, 2020; Moreno, 2020). Ahora bien, no debemos olvidar que en la situación actual estas tecnologías nos han demostrado ser de gran valía puesto que el uso adecuado de esta tecnología ofrece un recurso eficaz a nivel de trabajo, formación académica y ocio que antes no éramos capaces de visualizar en toda su amplitud (Carpio et al., 2020; Lozano et al, 2020).

\section{OBJETIVOS DE LA INVESTIGACIÓN}

El objetivo de este trabajo consiste en analizar los efectos que ha producido la pandemia provocada por el COVID-19 en jóvenes universitarios, en concreto: conocer los hábitos de uso de las nuevas tecnologías, el tiempo diario de uso dedicado al uso de internet y del teléfono móvil antes del COVID-19 y en la actualidad, las activi- 


\section{COVID-19: USO Y ABUSO DE INTERNET Y TELÉFONO MÓVIL EN ESTUDIANTES UNIVERSITARIOS}

dades para las que usan internet más frecuentemente y el uso problemático de las nuevas tecnologías en el ámbito académico, social y familiar.

\section{MUESTRA Y/O PARTICIPANTES}

En este estudio han participado 671 estudiantes de Grado de la Universidad de Jaén. Los participantes fueron seleccionados mediante un procedimiento incidental (o de conveniencia) intentando recoger datos de alumnos de diferentes grados y cursos de la Facultad de Humanidades y Ciencias de la Educación.

La participación en el estudio fue voluntaria y se garantizó la confidencialidad y el anonimato de sus respuestas. La distribución de la muestra en cuanto al sexo fue de 582 chicas (86,6\%) y 89 chicos (13,2\%), con edades comprendidas entre los 18 y los 42 años ( $M=20,63 ; S D=3,58)$. Respecto al curso, 383 estudiantes eran de $1^{\circ}$ curso (57\%), 288 estudiantes de $2^{\circ}$ curso (42,9\%). Finalmente, en cuanto al Grado que cursaban, 287 eran del Grado en Psicología (42,7\%), 319 del Grado de Educación Infantil (47,5 \%) y 65 estudiantes del Grado de Educación Social (9,7 \%). Para el cálculo de la representatividad en la muestra participante, se ha utilizado la fórmula para poblaciones con menos de 100.000 sujetos, con un nivel de confianza del 95\% y un error de estimación máximo de 4\%. Así pues, y considerando la titulación en que se encuentran matriculados los estudiantes universitarios objeto de estudio, se ha asegurado su representatividad para cada una de ellas, tomando como referencia los datos del alumnado matriculado en cada titulación en el curso 2020-2021.

\section{METODOLOGÍA E INSTRUMENTOS}

Para la recogida de los datos se adaptó el Cuestionario de Adicciones a las Nuevas Tecnologías (Oliva et al., 2012). En el cuestionario, además de recopilar información sociodemográfica de la muestra (sexo, edad, curso, titulación y disposición de internet en casa), se ha recogido información sobre los hábitos de uso de internet y teléfono móvil, en concreto: edad de inicio, nivel de uso, tiempo diario de uso dedicado antes de la pandemia y durante la misma y tipo de actividades para las que utilizan habitualmente internet. Finalmente se añadieron seis cuestiones que corresponden a situaciones en las que puede darse un uso problemático de internet y del teléfono móvil en el ámbito académico, social y familiar, similares a las utilizadas por Díaz-Vicario et al. (2019). En concreto, las situaciones eran si el uso de internet y el teléfono móvil provoca mayor distracción en clase, disminuye el tiempo de estudio y el tiempo invertido en otras actividades, reduce el tiempo presencial con amigos y familia y supone pasar mayor tiempo en soledad.

Esta investigación responde a un diseño metodológico cuantitativo, de carácter descriptivo y no experimental, con el fin de conocer los hábitos de uso de las nuevas tecnologías en estudiantes universitarios, así como las consecuencias de las mismas en momentos de pandemia. El cuestionario utilizado se adaptó a un formulario de Google Form, la administración se realizó durante el mes de febrero de 2021.

\section{RESULTADOS}

Con el objetivo de conocer los hábitos de uso de las nuevas tecnologías en los jóvenes en primer lugar, se consultó sobre la disposición de internet en casa, siendo un 99\% de la muestra los que disponían de ella frente a un $1 \%$ que contestaron que no. En cuanto a la edad de inicio, la edad media de inicio de internet es 11,06 años $(S . D .=3,27)$ y la edad de inicio del teléfono móvil 12,51 años (S.D.=4,86). Asimismo, se consultó a los jóvenes sobre el nivel de uso de estas tecnologías. Como podemos ver en la tabla 1, el $56 \%$ de la muestra señala tener un conocimiento avanzado de internet, seguido de un $29,5 \%$ que señalan tenerlo medio, un $12,5 \%$ experto y solo $1,8 \%$ se consideran principiantes en el uso de esta tecnología. En cuanto al uso del teléfono móvil, el 55,8 \% indican tener un conocimiento avanzado, un 30,4\% experto, un $12,6 \%$ medio y un $0,7 \%$ se consideran principiantes en esta tecnología. 
Tabla 1. Distribución de la muestra según su conocimiento en el uso de las nuevas tecnologías

\begin{tabular}{cccccc} 
& Nulo & Principiantes & Medio & Avanzado & Experto \\
\hline Internet & - & $12(1,8 \%)$ & $(198)$ & $(376) 56 \%$ & $(84) 12,5 \%$ \\
& & & $29,5 \%$ & & \\
Teléfono móvil & $2(0,3 \%)$ & $5(0,7 \%)$ & $(85)$ & $(375)$ & $(204)$ \\
& & & $12,6 \%$ & $55,8 \%$ & $30,4 \%$ \\
\hline
\end{tabular}

Otra variable analizada ha sido el número de horas que diariamente dedican al uso de internet y teléfono móvil, diferenciando entre el tiempo que dedicaban a ello antes del COVID-19 y en la actualidad. Como podemos ver en la tabla 2, se incrementa el porcentaje de estudiantes que usan internet más de 6 horas al día, pasando de un $14,3 \%$ a un $45,1 \%$, de igual manera ocurre con el uso del teléfono móvil, se incrementa el porcentaje de estudiantes que usan el teléfono más de 6 horas al día, pasando de un 21,3 \% a un 35,3\%. Para analizar si estos cambios resultaban significativos se presenta el valor del análisis de frecuencias a través de la prueba Chi-cuadrado y su nivel de significación, siendo en el caso de Internet: $\left[X^{2}(4, N=671)=350.15, p=0,000\right]$ y teléfono móvil X² $(4, N=666)=451.43, p=0,000]$.

Tabla 2. Tiempo diario de uso dedicado al uso de internet y teléfono móvil, antes del COVID y en la actualidad

\begin{tabular}{|c|c|c|c|c|c|c|c|}
\hline & & & \multirow[b]{2}{*}{ Ninguna } & \multirow[b]{2}{*}{$\begin{array}{c}\text { Menos } \\
\text { de } 1 \\
\text { hora }\end{array}$} & \multirow[b]{2}{*}{$\begin{array}{c}\text { Entre } \\
1-3 \\
\text { horas }\end{array}$} & \multirow[b]{2}{*}{$\begin{array}{c}\text { Entre } \\
3-6 \\
\text { horas }\end{array}$} & \multirow[b]{2}{*}{$\begin{array}{l}\text { Más de } \\
6 \text { horas }\end{array}$} \\
\hline & & & & & & & \\
\hline \multirow{4}{*}{ Internet } & Antes & $\mathrm{n}$ & 0,5 & 70 & 272 & 228 & 96 \\
\hline & & $\%$ & 0,7 & 10,4 & 40,5 & 33,9 & 14,3 \\
\hline & Actualidad & $\mathrm{n}$ & 3 & 21 & 110 & 234 & 303 \\
\hline & & $\%$ & 0,4 & 3,1 & 16,4 & 34,8 & 45,1 \\
\hline \multirow{4}{*}{$\begin{array}{l}\text { Teléfono } \\
\text { móvil }\end{array}$} & Antes & $\mathrm{n}$ & 0,7 & 18 & 196 & 307 & 143 \\
\hline & & $\%$ & 1 & 2,7 & 29,2 & 45,7 & 21,3 \\
\hline & Actualidad & $\mathrm{n}$ & 0 & 15 & 130 & 284 & 237 \\
\hline & & $\%$ & 0 & 2,2 & 19,3 & 42,3 & 35,3 \\
\hline
\end{tabular}

Otro de los objetivos de este trabajo ha sido conocer la frecuencia de uso de internet en actividades como redes sociales, correo electrónico, descargas, búsqueda de información y juegos online con y sin dinero. Tal y como podemos ver en la tabla 3, los datos más representativos indican que el $83 \%$ de los chicos y chicas utilizan todos los días las redes sociales y el $14,1 \%$ casi todos los días; un $57,6 \%$ de la muestra ha utilizado el correo electrónico todos los días y el 37,9\% casi todos los días. Respecto a la descarga de programas, películas o vide0s, el 45,8 \% alguna vez al mes. En cuanto a la realización de trabajos académicos el 57,3\% lo han utilizado todos los días y el $38,1 \%$ casi todos los días. Para la búsqueda de información sobre aficiones el $33 \%$ lo han utilizado todos los días y el $36 \%$ casi todos los días. En cuanto al juego online a través de internet sin dinero, un 46,3\% indican no haberlo utilizado nunca. Finalmente, casi la totalidad de la muestra, un 90,9\% señalan no haber jugado online a través de internet con dinero. 


\section{COVID-19: USO Y ABUSO DE INTERNET Y TELÉFONO MÓVIL EN ESTUDIANTES UNIVERSITARIOS}

Tabla 3 . Distribución de la muestra según las actividades para las que usan internet en la actualidad

\begin{tabular}{ccccccc}
\cline { 2 - 6 } & & Nunca & $\begin{array}{c}\text { Menos de } \\
1 \text { vez al } \\
\text { mes }\end{array}$ & $\begin{array}{c}\text { Alguna } \\
\text { vez al } \\
\text { mes }\end{array}$ & $\begin{array}{c}\text { Casi } \\
\text { todos los } \\
\text { días }\end{array}$ & $\begin{array}{c}\text { Todos } \\
\text { los días }\end{array}$ \\
\cline { 2 - 7 } $\begin{array}{c}\text { Uso de redes sociales, } \\
\text { mensajería... }\end{array}$ & $\mathrm{n}$ & $(1)$ & $(5)$ & $(11)$ & $(95)$ & $(558)$ \\
\hline $\begin{array}{c}\text { Utilizar el correo } \\
\text { electrónico }\end{array}$ & $\mathrm{n}$ & $(2)$ & $(3)$ & $(24)$ & $(255)$ & $(387)$ \\
\hline Descargar programas, & $\mathrm{n}$ & $0,3 \%$ & $0,4 \%$ & $3,6 \%$ & $37,9 \%$ & $57,6 \%$ \\
películas, videos... & $\%$ & $5,5 \%$ & $15,6 \%$ & $45,8 \%$ & $20,2 \%$ & $12,6 \%$ \\
\hline Trabajos académicos & $\mathrm{n}$ & 2 & $(2)$ & $(26)$ & $(256)$ & $(385)$ \\
& $\%$ & $0,3 \%$ & $0,3 \%$ & $3,9 \%$ & $38,1 \%$ & $57,3 \%$ \\
\hline Búsqueda de & $\mathrm{n}$ & $(5)$ & $(43)$ & $(159)$ & $(242)$ & $(222)$ \\
información & $\%$ & $0,7 \%$ & $6,4 \%$ & $23,7 \%$ & $36 \%$ & $33 \%$ \\
\hline Jugar online a través & $\mathrm{n}$ & $(311)$ & $(145)$ & $(108)$ & $(58)$ & $(49)$ \\
de internet sin dinero & $\%$ & $46,3 \%$ & $21,6 \%$ & $16,1 \%$ & $8,6 \%$ & $7,3 \%$ \\
\hline Jugar online a través & $\mathrm{n}$ & $(611)$ & $(27)$ & $(20)$ & $(3)$ & $(10)$ \\
de internet con dinero & $\%$ & $90,9 \%$ & $4 \%$ & $3 \%$ & $0,0,4 \%$ & $1,5 \%$ \\
\hline
\end{tabular}

Finalmente, se analizaron seis situaciones en las que puede darse un uso problemático en internet y teléfono móvil y que afectan al ámbito académico, social y familiar. En concreto, distracción en clase, tiempo de estudio y tiempo invertido en otras actividades como tiempo presencial con amigos y familia y soledad. Aunque las respuestas tenían cuatro posibles opciones (nada, poco, bastante y mucho) se decidió agruparlas en dos categorías: nada-poco y bastante-mucho, tal y como se presenta en la tabla 4, y analizar si se producían diferencias significativas en el uso de internet y del teléfono móvil entre los dos grupos de estudiantes.

Tabla 4. Distribución de la muestra según los resultados de las variables sobre uso problemático de internet y el teléfono móvil

\begin{tabular}{c|c|c|}
\cline { 2 - 3 } & Nada-Poco & Bastante-Mucho \\
\hline Distracción en clase & $370(55,1 \%)$ & $301(44,8 \%)$ \\
\hline Disminuir el tiempo de estudio & $388(57,7 \%)$ & $283(42,1 \%)$ \\
\hline $\begin{array}{c}\text { Disminuir el tiempo en otras } \\
\text { actividades }\end{array}$ & $453(67,4 \%)$ & $218(32,4 \%)$ \\
\hline $\begin{array}{c}\text { Disminuir tiempo presencial con } \\
\text { amigos }\end{array}$ & $629(93,6 \%)$ & $41(6,1 \%)$ \\
\hline $\begin{array}{c}\text { Disminuir tiempo presencial con la } \\
\text { familia }\end{array}$ & $569(84,6 \%)$ & $102(15,2 \%)$ \\
\hline Pasar más tiempo en soledad & $481(71,6 \%)$ & $190(28,3 \%)$ \\
\hline
\end{tabular}

Con respecto a la situación que implicaba la distracción en clase: el 55,1\% señalan que el uso del móvil e internet no les distrae en clase frente a un $44,8 \%$ de los jóvenes que afirman que el uso de internet y el teléfono móvil si les distrae en clase. En concreto, se han encontrado diferencias significativas en la variable uso de internet $t(667)=-10,77, p=, 000$ ( $M=33,43$ vs $M=40,95)$ y uso de teléfono móvil: $t(667)=-9,74, p=, 000$ ( $M=15,20$ vs $M=18,13$ ) entre ambos grupos.

En cuanto a disminuir el tiempo de estudio: el 57,7\% afirman que el uso de internet y el teléfono móvil no influye en su tiempo de estudio frente al $42,1 \%$ de los jóvenes que afirman haber reducido su tiempo de estudio. 
Se han encontrado diferencias significativas en la variable uso de internet $t(667)=-13,38, p=, 000$ ( $M=32,99$ vs $M=42,05)$ y uso de teléfono móvil: t(667)=-11,75, $p=, 000$ ( $M=15,06$ vs $M=18,52$ ) entre ambos grupos.

En referencia a la disminución del tiempo en otras actividades: el 67,4 \% informa que el uso de internet y del teléfono móvil no influye en el tiempo que dedican a otras actividades frente al 32,4 \% de los jóvenes que afirman que ha disminuido el tiempo que invierte en otras actividades Se han encontrado diferencias significativas en la variable uso de internet $t(667)=-12,15, p=, 000$ ( $M=33,94$ vs $M=42,77)$ y uso de teléfono móvil: t(667)=$9,55 p=, 000(M=15,53$ vs $M=18,58)$ entre ambos grupos.

En la consecuencia relativa a la disminución del tiempo presencial con los amigos tan solo un 6,1\% de los jóvenes afirman que ha reducido el tiempo que pasa presencialmente con los amigos frente al 93,6\% que informan que no reducen dicho tiempo. Se han encontrado diferencias significativas en la variable uso de internet t(668)=$6,15, p=, 000$ ( $M=36,24$ vs $M=45,63$ ) y uso de teléfono móvil: $t(668)=-5,99, p=, 000$ ( $M=16,28$ vs $M=20,17)$.

En referencia a la disminución del tiempo presencial con las familias, un 15,2 \% de los jóvenes afirman que han reducido el tiempo que pasa presencialmente con la familia por estar utilizando el teléfono móvil e internet, pero un $84,6 \%$ señalan que no. Se han encontrado diferencias significativas en la variable uso de internet $t(668)=-9,28, p=, 000$ ( $M=35,42$ vs $M=44,57)$ y uso de teléfono móvil: t(668)=-10,43, $p=, 000$ ( $M=15,87$ vs $M$ $=20,16)$ entre ambos grupos.

Por último, respecto al aumento del tiempo a solas, el $71,6 \%$ señalan que el uso de internet y el teléfono móvil no les supone pasar más tiempo a solas frente al 28,3\% de los jóvenes que confiesan que si. Se han encontrado diferencias significativas en la variable uso de internet $t(668)=-10,61, p=, 000(M=34,50$ vs $M=42,68)$ y uso de teléfono móvil: t(668)=-9,64, $p=, 000$ ( $M=15,61$ vs $M=18,81$ ) entre ambos grupos de estudiantes.

\section{DISCUSIÓN Y CONCLUSIONES}

Los resultados de este trabajo descriptivo nos aportan datos sobre los hábitos de uso de las nuevas tecnologías en jóvenes universitarios, así como los cambios que se han producido en la utilización de las mismas como consecuencia de las restricciones por la COVID-19 y las consecuencias o aspectos que han podido verse afectados por dicho uso.

Podemos señalar como la tendencia que se venía produciendo desde años atrás con respecto al incremento progresivo de internet en los hogares españoles se ha visto reforzada por la situación sobrevenida de pandemia. Así, según el INE (2020) en 2020 el 95.3\% de los hogares cuentan con acceso a Internet en casa. En este estudio el porcentaje se incrementa hasta el 99\%, lo cual es comprensible considerando que la muestra se compone de estudiantes universitarios que han necesitado dichos recursos durante este periodo.

Asimismo, los datos obtenidos también confirman la tendencia a la disminución en la edad de inicio en el uso de estas tecnologías. Esta disminución puede observarse al comparar los resultados obtenidos en el estudio de Oliva et al., (2012) con una media de edad de inicio de 15.08 años en internet y de 14.62 años en el uso del móvil frente a medias de 11,06 años y 12,5 años respectivamente en nuestro caso.

Con respecto al nivel de uso de estas tecnologías la muestra revela un buen dominio de las mismas, con un nivel que supera el avanzado en más del $68 \%$ en el caso de internet y más del $86 \%$ en el caso del teléfono móvil. Estos datos indican la rápida extensión de estas tecnologías entre los jóvenes a causa de las ventajas y oportunidades que representan a distintos niveles.

Es destacable el aumento significativo en el número de horas dedicadas al uso de internet y del teléfono móvil al comparar la situación previa a la pandemia y la situación actual. Según los datos obtenidos para Internet, antes del COVID más del 50\% de la muestra lo utilizaba menos de 3 horas diarias, mientras que en la actualidad el $45 \%$ lo utiliza más de 6 horas diarias. En cuanto al uso del móvil, aunque se parte de un nivel de uso mayor antes de la pandemia, también se produce un aumento significativo en el número de estudiantes que lo emplean más de 6 horas al día en la actualidad. Estos resultados coinciden con los obtenidos por GarcíaManglano et al. (2020) quienes encontraron que el incremento en la utilización de dispositivos digitales sobrepasaba las nueve horas de uso diario. 


\section{COVID-19: USO Y ABUSO DE INTERNET Y TELÉFONO MÓVIL EN ESTUDIANTES UNIVERSITARIOS}

En relación con las actividades para las que los estudiantes han empleado estos dispositivos durante este último periodo podemos destacar que dichas actividades se relacionan principalmente con sus necesidades académicas y socio-comunicativas. Estos datos coinciden tanto con los obtenidos en estudios previos a la pandemia (Cruces et al., 2016; Espuny, et al., 2011, Molero et al., 2014; Osorio et al., 2014) como con estudios más recientes, como el realizado por García-Manglano et al., (2020) donde se señala que, a pesar del aumento del uso durante el confinamiento, la utilización de los mismos ha sido principalmente para sus estudios o para comunicarse con amigos y familiares.

De estos resultados podemos concluir que el aumento de las tecnologías durante el confinamiento en los jóvenes universitarios no parece preocupante puesto que ha venido impuesto por las condiciones de la pandemia y se ha asociado a la utilización de estos medios en la impartición de la enseñanza online y en la sustitución de las relaciones y de la comunión cara a cara. En efecto, la pandemia ha supuesto una reclusión y un cambio de rutinas en la vida académica con la imposibilidad de asistencia física a clase en determinados momentos y en la vida social que se ha reflejado en la utilización de estos dispositivos como forma de aprender, de comunicarse y relacionarse.

En relación a los usos problemáticos de las nuevas tecnologías, los resultados obtenidos confirman que si bien, no son mayoritarios los estudiantes que presentan un uso excesivo de estas nuevas tecnologías, aquellos estudiantes que mas tiempo dedican a internet y al teléfono móvil muestran más problemas en el ámbito académico, dedicando menos tiempo al estudio y presentando problemas de distracción en clase, datos que coinciden con los obtenidos por Haddon y Vicent (2014) y Díaz-Vicario et al., (2019). Asimismo, los resultados indican que el mayor uso de estos dispositivos puede tener como consecuencia una disminución en el tiempo presencial con la familia y un mayor tiempo en soledad.

En cuanto a la dimensión social-relacional de estos jóvenes universitarios, el uso excesivo de internet y el teléfono móvil, puede afectar negativamente a las relaciones que tienen con su familia, creando conflictos a causa de utilizar estos dispositivos mientras realizan actividades en familia (Haddon y Vicent, 2014). Respecto a los amigos ocurre algo similar, sin embargo, los estudios señalan que no supone necesariamente empobrecimiento de las relaciones sociales, puesto que continuamente están conectados con amigos (De Almedia et al., 2014). El problema principal puede generarse debido a la situación de dependencia que presentan por la necesidad de mantenerse socialmente activos en cualquier momento del día, aunque muchos de ellos no son conscientes de ello (Ballesteros y Megías, 2015).

La importancia que estos recursos tecnológicos tienen en el desarrollo personal y social de los estudiantes hace necesario seguir avanzando y profundizando en su estudio para conocer a largo plazo el impacto tanto positivo como negativo que generan. También sería interesante replicar este estudio pasado un tiempo, cuando volvamos al estado de normalidad, con la finalidad de conocer si la eliminación de las restricciones lleva a que los jóvenes reduzcan el uso que hacen de las tecnologías o si esos nuevos hábitos de vida a los que nos ha obligado la pandemia se mantienen en el tiempo.

\section{REFERENCIAS BIBLIOGRÁFICAS}

Alonso, C. y Romero, E. (2017). El uso problemático de nuevas tecnologías en una muestra clínica de niños y adolescentes. Personalidad y problemas de conducta asociados, Actas Españolas Psiquiatría, 45(2), 62-70.

Arab, E y Díaz, A. (2015) Impacto de las redes sociales e internet en la adolescencia: aspectos positivos y negativos, Revista Médica Clínica Las Condes, 26(1), 07-13.

Ballesteros, J.C. y Megías, I. (2015). Jóvenes en la red: un selfie. Madrid: Centro Reina Sofía sobre Adolescencia y Juventud

Becoña, E. (2006). Adicción a nuevas tecnologías. La Coruña: Nova Galicia Edicions.

Besoli, G., Palomas, N. y Chamarro, A. (2018). Uso del móvil en padres, niños y adolescentes: Creencias acerca de sus riesgos y beneficios, Aloma, 36(1), 29-39. 
Carpio, M.V., García, M.C, Casanova, P.F y Cerezo, M.T, (2020). Efectos delconfinamiento por Covid-19 en el uso de internet, videojuegos y móvil enuniversitarios. Comunicación presentada en el XIII Congreso internacional de psicología clínica (Noviembre, 2020).

Cerezo, M.T, Casanova, P.F., García, M.C. y Carpio, M.V (2020). Uso/abuso de internet, videojuegos y móvil y variables de personalidad en universitarios durante el confinamiento. Comunicación presentada en el XIII Congreso internacional de psicología clínica (Noviembre, 2020).

Cruces, S., Guil, R., Sánchez, N. y Pereira, J.A. (2016). Consumo de nuevas tecnologías y factores de personalidad en estudiantes universitarios, Revista de Comunicación y Ciudadanía Digital, 5(2), 2003-228.

De Almedia, A.N., Delicado, A., De Almedia-Alves, N. y Carvalho, T. (2014). Internet, children and space: Revisiting generational attributes and boundaries. New Media \& Society, 17(9), 1436-1453.

Díaz- Vicario, A., Mercader, C. y Gairín, J. (2019). Uso problemático de las TIC en adolescentes, Revista Electrónica de Investigación Educativa, Vol 21, 2019/e07

Echeburúa, E., Labrador, F. J. y Becoña, E. (2009). Adicción a las nuevas tecnologías en adolescentes y jóvenes. Madrid: Pirámide.

Espuny, C., González, J., Lleixá, M., y Gisbert, M. (2011). Actitudes y expectativas del uso educativo de las redes sociales en los alumnos universitarios, Revista de Universidad y Sociedad del Conocimiento, 8(1), 171-185.

García-Manglano, J., Sábada, C. y López, C. (2020). El bienestar personal y el uso de la tecnología en confinamiento. Obra Social la Caixa https://observatoriosociallacaixa.org/-/el-bienestar-personal-y-el-uso-delatecnologia-en-confinamiento

Haddon, L. y Vicent, J. (2014). European children and their carers' understanding of use, risks and safety issues relating to convergent mobile media. Reporte D4.1. Milán: Unicatt.

Heyman, A. (2014). The influence of Social Networks on students' performance. Journal of Emerging Trends in Computing and Information Sciencies, 5(3), 200-205.

Instituto Nacional de Estadística (2020). Encuesta sobre equipamiento y uso de tecnologíasde información y comunicación en los hogares. Madrid: INE.

Labrador, F. J. y Villadangos, S. (2009). Adicción a nuevas tecnologías en adolescentes y jóvenes. En E. Echeburúa, F. J. Labrador y E. Becoña (Coords.), Adicción a las nuevas tecnologías en adolescentes y jóvenes (pp. 45-75). Madrid: Pirámide.

Lozano- Díaz, A., Fernández-Prados, J.S., Figueredo, V. y Martínez, A.M (2020). Impactos del confinamiento por el COVID-19 entre universitarios: Satisfacción Vital, Resiliencia y Capital Social Online, International Journal of Sociology of Education, Special Issue 2020, 79-104

Martínez de Moretín, J.I., Cortés, A., Medrano, C. y Apodaca, P. (2014). Internet use and parental mediation: A cross-cultural study, Computers y Education, 70, 212-221.

Molero, M., Martos, A., Cardila, F., Barrgán, A.B., Pérez-Fuentes, M.C., Gazquez; J.J. y Gil, J. (2014). Uso de internet y redes sociales en estudiantes universitarios, European Journal of Child development, 2(3), 81-96.

Moreno, C. (04 de abril de 2020). Juego, drogas y pantallas: así se tratan las adicciones en periodo de confinamiento. https://www.elsaltodiario.com/coronavirus/juegodrogas- pantallas-asi-tratan-adicciones-periodoconfinamiento

Muñoz-Miralles, R. Ortega-González, R., Batalla- Martínez, C., López-Morón, M.R. Manresa, J.M. y ToránMonserrat, P. (2014). Acceso y uso de nuevas tecnologías entre los jóvenes de educación secundaria, implicaciones en salud. Estudio JOITIC, Atención Primaria, 46(2), 77-88.

Muñoz-Rivas, M., Fernández, L. y Gámez-Guadix, M. (2009). Adicción y abuso del teléfono móvil. En E. Echeburúa, F. J. Labrador y E. Becoña (Ed.), Adicción a las nuevas tecnologías en adolescentes y jóvenes (pp. 131-149). Madrid: Pirámide.

Oliva, A., Hidalgo, M.V., Moreno, C., Jiménez, L., Jimémez, A., Antolin, L. y Ramos, P. (2012). Uso y riesgo de adicciones a las nuevas tecnologías entre adolescentes y jóvenes andaluces. Ed. Aguaclara. 


\section{COVID-19: USO Y ABUSO DE INTERNET Y TELÉFONO MÓVIL EN ESTUDIANTES UNIVERSITARIOS}

Orte, C. y Nevot-Caldentey, LI. (2020). Manual de recursos para familias en confinamiento 2020. Grupo de Investigación y Formación Educativa y Social (GIFES). Ed. Octaedro

Orte, C., Ballester, LI. y Nevot-Caldentey (2020). Apoyo familiar ante el COVID-19 en España. file:///Users/uja/Downloads/297-Preprint\%20Text-337-1-10-20200502.pdf Osorio et al. 2010).

Osorio, M.J., Molero, M.M., Pérez, M.C. y Mercader, I. (2014). Redes sociales en internet y consecuencias de su uso en estudiantes universitarios, International Journal of Developmental and Educational Psychology, 1(3), 585-592.

Pérez-Díaz, V. y Rodríguez, J. C. (2008). La adolescencia, sus vulnerabilidades y las nuevas tecnologías de la información y la comunicación. Madrid: Fundación Vodafone España.

Pintado, T. y Sánchez, J. (2012). Técnicas de comunicación en la Web 2.0. En T. Pintado y J. Sánchez (Ed.). Nuevas tendencias en comunicación (pp. 107-132) Madrid: ESIC Editorial

Torres- Diaz, J.C., Duart, J.M., Gómez-Alvarado, H.F., Marín-Gutierrez, I. y Segarra-Faggioni, V. (2015). Usos de Internet y éxito académico en estudiantes universitarios, Comunicar,48, 61-70. 